\title{
ACID PRODUCTION AT PARTIAL OXYGEN TENSION AND UNDER AEROBIC CONDITIONS BY A BACILLUS OF THE TYPHOID DYSENTERY GROUP
}

\author{
Wade W. Oliver and Orman C. Pering \\ From the Department of Bacteriology, Hoagland Laboratory, Long Island College \\ Hospital, Brooklyn
}

This article deals with the fermentative reactions of a bacillus from the feces of a case of dysentery. Morphologically and culturally, the bacillus shows the characteristics of the dysentery bacillus. It is a short, nonmotile, gram-negative rod, with a typhoidlike growth on plain agar and gelatin, producing a clouding of broth with a small amount of deposit at the end of 3-5 days' incubation at 37 C. Milk is not coagulated and after a short initial acidity, the milk becomes more alkaline, as shown by a deepening of the blue color of the litmus. Gelatin is not liquefied and no gas is produced in carbohydrate mediums.

Four days after isolation, the fermentative characters of the organism were tested in sugar broths $(+1$ meat extract broth, previously rendered sugar free by inoculation with $B$. coli, to which were added the respective sugars, in approximately $1 \%$ amounts, and sufficient litmus to impart a definite blue color to the solution). At the end of 48 hours' incubation at $37 \mathrm{C}$. acid production was marked in dextrose. In mannite and saccharose there was a trace of acid, with partial reduction of litmus. After 4 days' incubation at $37 \mathrm{C}$. the dextrose broth was still markedly acid, but the reaction in mannite and saccharose had become alkaline. At the end of 9 days' incubation the dextrose tube alone showed acid production. Lactose, maltose, and dextrin remained alkaline. A second series of sugar broths inoculated from a plain agar aerobic slant, which was the second transplant on plain agar after the organism was obtained in pure culture, showed acid production in dextrose and alkali in lactose, maltose, mannite, saccharose, and dextrin, after 48 hours' incubation.

After three transfers on aerobic plain agar $(+1)$ slants, the organism was grown on plain agar $(+1)$ at partial oxygen tension by connecting it and incubating it with a slant inoculated with $B$. subtilis. ${ }^{1}$ The 48-hour growth at partial tension was marked but not quite so pronounced as on plain agar incubated aerobically. After continuous incubation on plain agar at partial tension for 2 days, transplants were made to slants of litmus sugar-ascitic Martin agar. ${ }^{2}$ The medium was prepared as follows: sodium phosphate agar, $0.5 \%$ acid to phenolphthalein as recommended by W. Blair M. Martin, was rendered sugar

Received for publication Jan. 2, 1918.

1 Wherry and Oliver: Jour. Infect. Dis., 1916, 19, p. 288, and 1917, 20, p. 28.

2 W. Blair M. Martin: Jour. Path. and Bacteriol., 1911, 15, p. 76. 
free by inoculation with B. coli. After filtering and heating, ascitic fluid was added in the proportion of about one of fluid to four of the agar, then the sugars, to a concentration of about $1 \%$, and then sufficient litmus to color the medium. The tubes were slanted.

On 24 hour incubation at partial oxygen tension, acid production in dextrose was marked, but lactose, galactose, maltose, mannite, levulose, and saccharose remained alkaline. On further incubation at partial tension for 48 hours not only did the dextrose slant show marked acid production, but likewise in the other sugars marked acid reaction had appeared, which persisted.

These findings were so unexpected that the partial tension sugar agars were examined microscopically with Gram's stain and were also plated out in plain agar to test the suspicion that we were working with a mixed culture. The plates showed a pure culture of a gram-negative rod which corresponded morphologically and culturally with the organism described as isolated from the stool.

After 3 days' incubation at 37 C. transplants were made to a second series of litmus sugar-ascitic Martin agar slants which were made at the same time and in a manner identical with those of the first series. Transplants to the sugar agars were made from the respective sugar agars of Series 1, namely, from dextrose to dextrose, mannite to mannite, etc. This second series (Series 2) was made partial tension in the same manner as the first and was then incubated at $37 \mathrm{C}$. Transplants (Series 3) were also made from partial tension sugars of the first series to respective sugar litmus broths $(+1)$ (Series 3 ) which were incubated aerobically at $37 \mathrm{C}$.

Forty-eight hours later, Series 2 (that is, the partial tension litmus sugarascitic Martin agar slants) showed marked acid production in dextrose, lactose, galactose, maltose, mannite, levulose, and saccharose. The aerobic sugar broth series (Series 3), on the other hand, showed acid production only in dextrose, thus corresponding with the original reactions obtained in aerobic sugar broths, when first tested after isolation from the stool. Thro advises against the use of ascitic fluid when testing the variability of micro-organisms on the carbohydrates, because he found that different samples of ascitic fluid vary in the amount of fermentable sugars that they contain. To overcome the objection that possibly the acid production obtained at partial tension in a variety of sugars might be due to the use of ascitic fluid in the medium, ${ }^{3}$ transplants were made from the partial tension Series 2 to slants of sugar free agar $(+1)$ to which respective sugars were added in a concentration of about $1 \%$. Likewise, the same procedure was employed as regards the aerobic Series 3, in each case transfers being made from the respective sugars. Again, identical results to those described were obtained, that is, under aerobic conditions, dextrose alone was fermented, whereas under conditions of partial oxygen tension acid production occurred not only in dextrose, but in lactose, galactose, maltose, mannite, levulose, and saccharose.

\section{DISCUSSION}

One of the most intensive studies of the influence of environment on the characters of micro-organisms, especially of the colon group, is that by Peckham. ${ }^{4}$ By modifying the environment she was able to exalt in some cases the activity of one function and in other cases to

s Thro, W. C.: Jour. Infect. Dis., 1915, 17, p. 227.

- Jour. Exper. Med., 1897, 2, p. 550. 
depress or even extinguish certain biologic traits. By a series of cultural experiments, for instance, she was able to force B. typhosus to produce indol.

Twort, ${ }^{5}$ in a study of bacterial variations, claimed that by prolonged cultivation on saccharose of different strains of the typhoid, the paratyphoid, and dysentery bacilli, they all acquired the property of fermenting saccharose. After repeated transfers on saccharose the dysentery bacillus was able to produce acid from this sugar in 24 hours. It required 2 years of constant growth on lactose for Twort to produce a lactose-fermenting variant of $\mathrm{B}$. typhosus.

In the case of an organism studied by Klotz, ${ }^{6}$ belonging to the colon group isolated from water, which did not ferment lactose or saccharose when first isolated, repeated transfers into lactose and saccharose broth, lead finally to the acquisition by the organism of the power of fermenting these sugars, with acid and gas production.

In regard to the experimental inhibition of fermentative activities, Herter, ${ }^{7}$ for instance, has shown that the fermentative activities of B. coli are considerably inhibited by the presence of sodium benzoate in weak glucose broth, whereas other biologic activities of the bacillus are but slightly, if at all, impaired. Sodium benzoate $(0.1 \%)$ in dextrose broth only slightly or moderately inhibits the growth of $\mathrm{B}$. coli, but gas production may, however, be considerably inhibited. Likewise, Penfold ${ }^{8}$ found that sodium acetate inhibits the fermentative activities of B. coli, B. enteritidis and B. paratyphi, probably by inhibition or destruction of the enzyme invertase, as evidenced by the fact that although gas formation in the sugar was diminished and finally totally disappeared, yet the bacillus was still capable of producing gas from the corresponding alcohols. Smirnow ${ }^{9}$ reports that continuous growth of B. coli in $3 \%$ glucose broth lead, in the case of 3 of 7 strains studied by him, to a complete inhibition of both acid and gas production in the following sugars: glucose, lactose, maltose, saccharose, dextrin, and mannite. In 2 other strains, glucose varied the amount of acid and gas production, with an occasional complete inhibition in some of the sugars.

A consideration of the foregoing work reveals what may be termed induced variations in the biologic activities of bacteria, especially as

5 Proc. Roy. Soc., B., 1907, 79, p. 329.

6 Jour. Infect. Dis., 1906, Sup. 2, p. 35.

7 Jour. Biol. Chem., 1909, 7, p. 59.

8 Brit. Med. Jour., 1911, Sup. 2, p. 363.

\& Jourr. Bacteriol., 1916, 1, p. 385. 
regards gas production and acid production. The results detailed in our communication possibly are capable of a different interpretation.

Wherry and Oliver ${ }^{1}$ have published observations which tend to establish the generalization that many bacterial as well as animal endoparasites become adapted to a tension of oxygen below the atmospheric. Within the body organisms, as a general rule, multiply and exhibit their characteristic activities under conditions of lowered oxygen tension. Corroborative evidence to this effect is supplied by our observations that the isolation and cultivation on laboratory mediums of such organisms as the meningococcus, gonococcus, certain strains of streptococci, etc., can best, and in some cases, only be effected by growing these organisms under conditions of partial oxygen tension. Admittedly, the culture mediums we employ in the laboratory are at best a poor substitute for the habitat that bacteria find in the body. However, the more closely we simulate body conditions in the test tube or flask, the more accurately, it would seem, will we be able to demonstrate in cultures the biologic activities that micro-organisms exercise within the body. We would suggest that possibly, in the case of the organism described in the present communication, the production of acid in dextrose, lactose, galactose, maltose, mannite, levulose, and saccharose, under conditions of partial oxygen tension, whereas under aerobic conditions, permanent acid production occurred only in glucose, took place because the organisms grown at diminished oxygen tension were supplied with conditions more closely simulating those encountered in their habitat in the body. Viewed in this light, we would be dealing not with an example of induced variation in a biologic character.

In conclusion, it may be pointed out that in the case of the organism studied in this paper, we seem to be dealing with certain enzymes which function only under an oxygen tension below the atmospheric.

\section{SUMMARY}

An organism, culturally of the typhoid-dysentery group, isolated from the feces in a case of dysentery, when grown under aerobic conditions, produced persistent acid from dextrose alone, whereas, when grown at diminished oxygen tension, acid was produced in dextrose, lactose, galactose, maltose, mannite, levulose, and saccharose. 